\title{
Breast cancer treatment in Hungary-first step into molecular medicine
}

István Láng

Received: 8 November 2012 / Accepted: 11 December 2012 / Published online: 17 January 2013

(C) Springer-Verlag Wien 2012

As new Section Editor for breast cancer of MEMO, this is my short introduction about breast cancer care in Hungary.

Breast cancer is responsible for the death of around 400,000 women per year worldwide with about 1,200,000 new cases per year. In industrialized countries, it is the number one cancer killer among women. In Hungary where I have been working at the National Institute of Oncology, there were 7,368 new breast cancer patients and 2,040 deaths in 2010.

In order to reduce breast cancer mortality in Hungary, population based mammography screening has been implemented for women between 45 and 65 years. Unfortunately its efficiency is reduced by insufficient participation of the invited women: instead of the desired $70 \%$ or more it is only about $50 \%$ at present, although health authorities, doctors, and patient advocacy organizations do their best to increase participation.

Apart from healthy life style and regular screening, another tool to reduce breast cancer mortality is more efficient treatment. This can be facilitated by offering breast cancer care for women in specialized comprehensive cancer centers, where the "critical mass" of patients ensures sufficient experience, and multidisciplinary cooperation of experts is possible in the field of diagnostics, pathology, surgical, radiation, and medical therapy.

At the National Institute of Oncology in Budapest, all these requirements are fulfilled, and the organization of further Comprehensive Breast Cancer Units are under way in the country. In Hungary, breast cancer care is free

\footnotetext{
Prof. Dr. I. Láng $(\bowtie)$
}

Department for Medical Oncology and Clinical Pharmacology “B”, National Institute for Oncology, Ráth György u. 7-9,

1122 Budapest, Hungary

e-mail: lang@oncol.hu

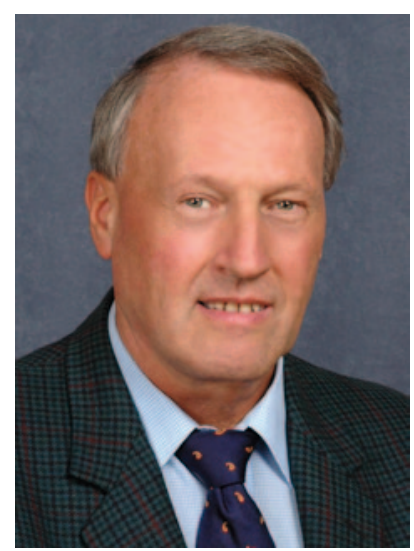

Istvan Láng

for all insured patients, and is financed by the National Health Insurance Company.

Medical oncological therapy for breast cancer is becoming more and more expensive by the introduction of high tech targeted agents. Nowadays, we witness another paradigm change in the medical oncological treatment of breast cancer.

During the first era, the choice of medical therapy was based on classical pathological findings like tumor size, number of metastatic regional nodes, histological type, grade, hormone receptors, etc.

In the next era, breast cancer therapy was based on the molecular pathological discoveries of the eighties resulting in new targeted agents, like trastuzumab and lapatinib, which were introduced during the nineties.

The next stage was the revolution of genomic profiling resulting in new classification of breast cancers according to their genetic signature, and the development of genomic diagnostic tools offering prognostic and predictive evaluation of individual tumors beyond the traditional methods.

The present paradigm is characterized by integration of molecular pathology and genomic profiling, making individually tailored therapy possible. One of our most important tasks is to promote the use of modern molecular pathology and genomic profiling, and by integrating them making personalized tailored therapy possible for more and more breast cancer patients.

In order to further decrease breast cancer mortality in Europe, breast cancer care should develop in certain important directions in the near future. These are:

- Increasing the number of early-detected breast cancers by appropriate participation of women in population based mammography screening.

- Organising breast cancer care in dedicated breast cancer centers where the sufficient number of patients 
(critical mass) and the availability of multidisciplinary oncoteam and modern technology can ensure highlevel comprehensive breast cancer care.

- Increasing the use of molecular pathology and genomic profiling for the determination of personalized, tailored treatment for each individual patient.
Our aim is that memo breast cancer publications should reflect and serve these goals in order to help better breast cancer care in Europe. 